\title{
DUPLICATION OF ROOM SQUARES
}

\author{
W. D. WALLIS \\ (Received 18 September 1970) \\ To Professor T. G. Room on his 70th birthday \\ Communicated by G. Szekeres
}

\section{Room squares}

A Room square $\mathscr{R}$ of order $2 n$ is a way of arranging $2 n$ objects (usually $1,2, \cdots, 2 n)$ in a square array $\mathscr{R}$ of side $2 n-1$ so that:

(i) every cell of the array is empty or contains two objects;

(ii) each unordered pair of objects occurs once in $\mathscr{R}$;

(iii) every row and column of $\mathscr{R}$ contains one copy of each object.

In his original note [6], Room showed that there are squares of orders 2 and 8 , but not of orders 4 and 6 . It is known that Room squares exist of every order $2^{2 k+1}, k$ integral [1], of every order $2 n=p^{r}+1$ where $p^{r}$ is a prime power other than a Fermat prime (of type $2^{2^{s}}+1$ ) [4], and of every order from 10 to 48 [8]. Moreover, if there are squares of orders $2 m$ and $2 n$, then there is a square of order $(2 m-1)(2 n-1)+1$ [9].

In this paper we prove that

If $2 n-1=\prod p_{i}^{r_{i}}$, where each $p_{i}$ is a prime congruent to 3 modulo 4 and no $p_{i}^{r_{i}}$ is $3,3^{2}$, or $3^{4}$, then there is a Room square of order $4 n$.

This provides apparently new Room squares of orders 56,88 and 96 (leaving six orders less than 100 undecided, namely $52,58,66,76,86$ and 94) and seven new squares of orders between 100 and 500 .

We consider two Room squares to be isomorphic if one can be obtained from the other by interchanging two rows, interchanging two columns, interchanging two objects, or any sequence of these operations. We say a square of order $2 n$ is standardized if it has the pair $\{i, 2 n\}$ in its $i$ th diagonal position; obviously any Room square is isomorphic to a standardized one.

The incidence matrix of a Room square $\mathscr{R}$ of order $2 n$ will mean the square matrix of size $2 n-1$ which has 1 in its $(i, j)$ position when $\mathscr{R}$ has an entry in that position and -1 otherwise. A standardized Room square will be called skew-type if its incidence matrix is $I+S$, where $S$ is a skew-symmetric matrix; and an 
arbitrary Room square is skew-type if it is isomorphic to a skew-type standardized square.

\section{Concerning Latin squares}

A Latin square of order $r$ is a square array of size $r$ on $r$ symbols (usually $1,2, \cdots, r$ ) such that every symbol occurs once in each row and each column. Given arrays $L=\left(l_{i j}\right)$ and $M=\left(m_{i j}\right)$ of the same size, we will write $(L, M)$ for the array whose $(i, j)$ element is the ordered pair $\left(l_{i j}, m_{i j}\right)$. In this notation two Latin squares $L$ and $M$ of the same order are called orthogonal if $(L, M)$ has no two entries the same.

THEOREM 1. (i) A Room square $\mathscr{R}$ of order $2 n$ is equivalent to a pair of symmetric Latin squares $R_{1}$ and $R_{2}$ of order $2 n$, each having constant diagonal $(0,0, \cdots, 0)$, such that $\left(R_{1}, R_{2}\right)$ has no two entries the same above the main diagonal.

(ii) If $\mathscr{R}$ is standardized then $R_{1}$ and $R_{2}$ each have last column $(1,2, \cdots, 2 n-1,0)$.

(iii) If the standardized Room square is skew-type then $\left(R_{1}, R_{2}\right)$ and $\left(R_{2}, R_{1}\right)$ have no common entries above the main diagonal except for $(1,1)$, $(2,2), \cdots(2 n-1,2 n-1)$, which appear in the last column of each.

Proof. (i) Given a Room square $\mathscr{R}$ on the objects $1,2, \cdots 2 n$, write $r_{i j}$ for the number of the row and $c_{i j}$ for the number of the column which contain the pair $\{i, j\}$. Write $r_{i i}=c_{i i}=0$, and write $R_{1}=\left(r_{i j}\right), R_{2}=\left(c_{i j}\right)$. It follows immediately from the definition of Room squares that $R_{1}$ and $R_{2}$ are symmetric Latin squares. They have the required diagonal. If the entry $(a, b)$ occurs in the $(i, j)$ position of $\left(R_{1}, R_{2}\right)$ this means $\{i, j\}$ lies in row $a$ and column $b$ of $\mathscr{R}$ and consequently $(a, b)$ cannot occur elsewhere in $\left(R_{1}, R_{2}\right)$ except in the $(j, i)$ position.

Given two Latin squares of the specified type, this construction can be reversed to give the Room square.

(ii) is obvious.

(iii) follows because entry $(i, j)$ occurs above the diagonal in $\left(R_{1}, R_{2}\right)$ if and only if the incidence matrix of $\mathscr{R}$ has 1 in its $(i, j)$ position, and because Latin squares $R_{2}$ and $R_{1}$ correspond via part (i) to the transpose of $\mathscr{R}$.

We will call the $R_{1}$ and $R_{2}$ which we have constructed the row and column Latin squares of $\mathscr{R}$ respectively.

Part (i) of Theorem 1 could be deduced from the facts that both Latin squares and Room squares are related to loops [3, Chapters 6, 10].

\section{Duplication of skew-type Room squares}

In this section we give a construction for a Room square twice the order of a given skew-type square. 
Suppose $\mathscr{R}$ is a skew-type Room square of order $2 n$. We may assume $\mathscr{R}$ to be standardized. Write $R_{1}$ and $R_{2}$ for the row and column Latin squares of $\mathscr{R}$. Suppose further that there are orthogonal Latin squares $L_{1}$ and $L_{2}$ of order $2 n$, each having last column $(1,2, \cdots, 2 n)$. Write $M_{\alpha}$ to mean the square $L_{\alpha}$ with each entry increased by $2 n-1$. Now write

$$
Q=\begin{array}{ll}
R_{1} & M_{1} \\
M_{1}^{T} & R_{2}
\end{array} \quad S_{2}=\begin{array}{ll}
R_{2} & M_{2} \\
M_{2}^{T} & R_{1}
\end{array}
$$

and write $S_{1}$ for the array obtained from $Q$ by interchanging rows $2 n$ and $4 n$ and interchanging columns $2 n$ and $4 n$. Then $\left(S_{1}, S_{2}\right)$ is as shown, where the notations are that $m_{i}^{\alpha}$ is the $(2 n, i)$ element of $M_{\alpha}$ and that $\hat{X}$ means the array $X$ with the last row and column deleted.

$S_{1}$ and $S_{2}$ are symmetric Latin squares of order $4 n$ with constant diagonal $(0,0, \cdots, 0)$. In the event of no duplications above the diagonal in $\left(S_{1}, S_{2}\right)$ they will imply the existence of a Room square of order $4 n$, by Theorem 1 .

The entries above the diagonal in $\left(S_{1}, S_{2}\right)$ are of three types:

(a) both elements less than $2 n$ : we have all the entries above the diagonal in $\left(\hat{R}_{1}, \hat{R}_{2}\right)$ and $\left(\hat{R}_{2}, \hat{R}_{1}\right)$ once each. By Theorem $2($ iii) these include no duplications.

(b) both elements at least $2 n$ : we have certain of the entries from $\left(M_{1}, M_{2}\right)$ (not including the entry $(4 n-1,4 n-1)$ which was in the $2 n$th row and column of $\left.\left(M_{1}, M_{2}\right)\right)$ and also $(4 n-1,4 n-1)$. As $M_{1}$ and $M_{2}$ are orthogonal, these include no duplications.

(c) one element less than $2 n$, and one not: we have all the $(i, 2 n+i-1)$, $\left(i, m_{i}^{2}\right),(2 n+i-1, i)$ and $\left(m_{i}^{1}, i\right)$ for $i=1,2, \cdots, 2 n-1$. These will include duplications if and only if $m_{i}^{1}=2 n+i-1$ or $m_{i}^{2}=2 n+i-1$ for some $i \leqq 2 n-1$; in terms of the original squares $L_{1}$ and $L_{2}$, if and only if the $i$ th element of the last row of $L_{1}$ or $L_{2}$ is $i$ for some $i \neq 2 n$.

Clearly, no duplications occur between the different types.

From [2] there are two orthogonal Latin squares of order $2 n$ provided $2 n \neq 2$ or 6 . Suppose we have two such Latin squares. By proper labelling of the entries and ordering of the columns, we can assure that both squares have last column $(1,2, \cdots, 2 n)$, and that the last row is $(1,2, \cdots, 2 n)$ in one and $\left(a_{1}, a_{2}, \cdots, a_{2 n-1}, 2 n\right)$ in the other where $a_{i} \neq i$. By Theorem 2.2 of Chapter 6 of [7] we can find $b_{1}, b_{2}, \cdots, b_{2 n-1}$ such that $i \neq b_{i} \neq a_{i}$. Re-order the columns of the squares so that column $i$ becomes column $b_{i}$. If we take these squares as $L_{1}$ and $L_{2}$, there will be no duplications in the elements of type (c).

Summarising, we have the following result.

THEOREM 2. If there is a skew-type Room square of order $2 n>2$, then there is a Room square of order $4 n$. 


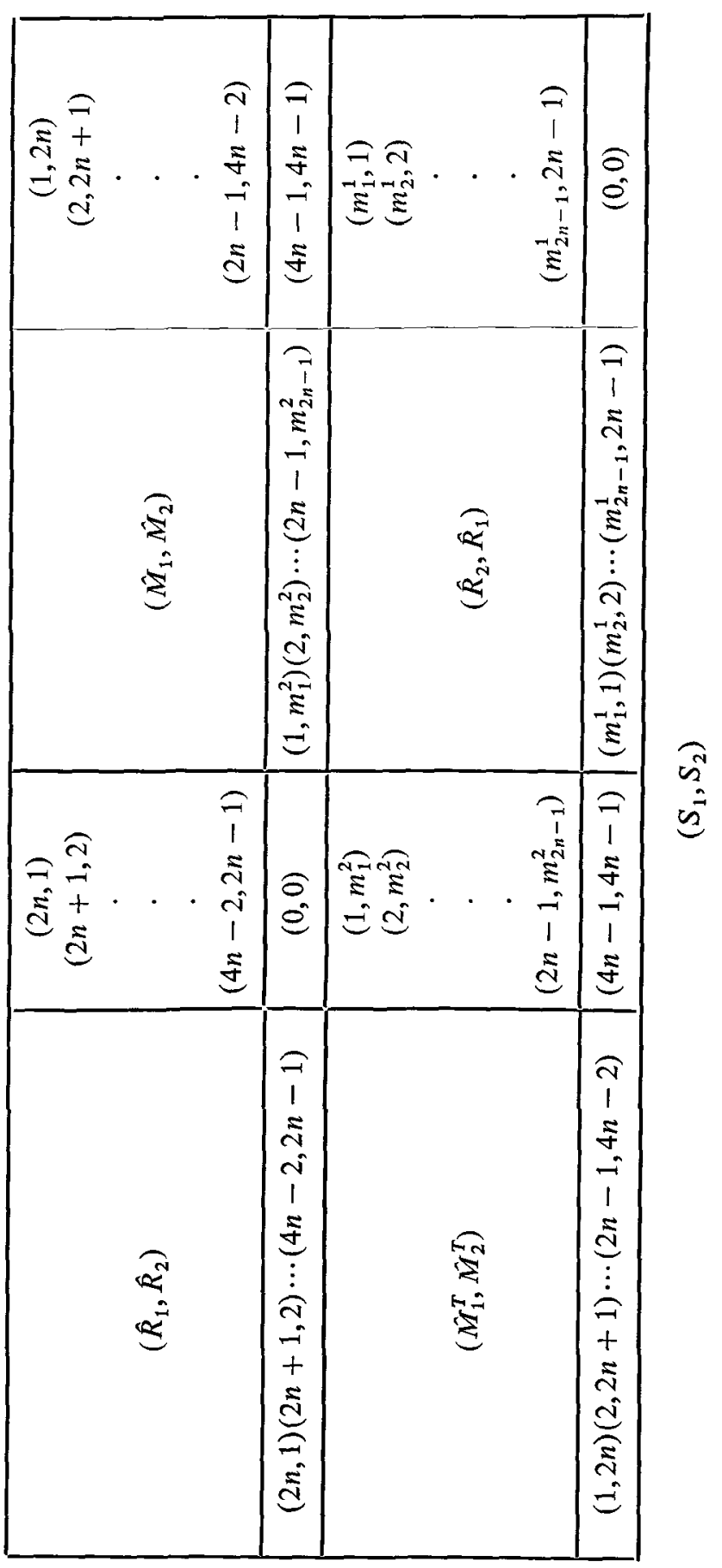




\section{The Mullin-Nemeth squares}

Suppose $n$ is even and $2 n-1$ is a prime power greater than 3. Choose a generator $x$ of the multiplicative group of non-zero elements in the Galois field $G F(2 n-1)$, and write the elements of $G F(2 n-1)$ as $g_{1}, g_{2}, \cdots, g_{2 n-1}$, where $g_{1}=0$ and $g_{i}=x^{i-2}$ otherwise. A Room square of order $2 n$ may be formed in the following way:

(i) the $(i, i)$ cell contains $\{i, 2 n\}$;

(ii) the $(1, k)$ cell contains $\{2 p, 2 p+1\}$ when $g_{k}=g_{2 p}+g_{2 p+1}, p=1,2, \cdots$ $n-1$; if $g_{k}$ cannot be expressed in this way, then cell $(1, k)$ is empty;

(iii) the $(i, j)$ cell depends on the $(1, k)$ cell where $g_{k}=g_{j}-g_{i}$. If the $(1, k)$ cell is empty then the $(i, j)$ cell is empty; if the $(1, k)$ cell contains $\{2 p, 2 p+1\}$ then the $(i, j)$ cell contains $\{q, r\}$, defined by

$$
g_{q}=g_{2 p}+g_{i}, q_{r}=g_{2 p+1}+g_{i} .
$$

This is the construction given by Mullin and Nemeth in [4]. (For proof of the Room property see [4] and [5].) Its incidence matrix A has diagonal elements $a_{i i}=1$; if $i \neq j, a_{i j}=1$ if and only if $g_{j}-g_{i}$ is $(x+1)$ times an even power of $x$. If $a_{i j}=a_{j i}=1$ then both $(x+1)^{-1}\left(g_{j}-g_{i}\right)$ and $(x+1)^{-1}\left(g_{i}-g_{j}\right)$ are quadratic elements in $G F(2 n-1)$, which is impossible as $2 n-1 \equiv 3(\bmod 4)$. So the MullinNemeth construction gives a skew-type Room square.

COROLLARY 3. There is a skew-type Room square of order $2 n=p^{r}+1>4$ whenever $p^{r}$ is a prime power congruent to 3 modulo 4 .

\section{A multiplication theorem}

THEOREM 4. If there are Room squares $\mathscr{M}$ and $\mathscr{N}$ of orders $2 m$ and $2 n$, then there is a Room square $\mathscr{R}$ of order $(2 m-1)(2 n-1)+1$. If $\mathscr{M}$ and $\mathscr{N}$ are skew-type, then so is $\mathscr{R}$.

PROOF. If $n=1$ there is nothing to prove. So we assume $n>1$, whence there exist a pair of orthogonal Latin squares of order $2 n-1$; call them $L_{1}$ and $L_{2}$. Assume that $\mathscr{M}$ and $\mathscr{N}$ are standardized; write the row and column Latin squares of $\mathscr{M}$ as $M_{1}=\left(a_{i j}^{1}\right)$ and $M_{2}=\left(a_{i j}^{2}\right)$, and those of $\mathscr{N}$ as $N_{1}$ and $N_{2}$. Again $\hat{N}_{\alpha}$ is $N_{\alpha}$ with the last row and column deleted.

For convenience we will write the numbers from 1 to $(2 m-1)(2 n-1)$ in the form $x_{y}$, where

$$
x_{y}=x+(y-1)(2 n-1), 0<x<2 n, 0<y<2 m .
$$

(Define $0_{y}$ as 0 .) If $A$ is any of the matrices $\hat{N}_{1}, \hat{N}_{2}, L_{1}, L_{2}$, we will write $A(y)$ to mean the matrix A with each entry $x$ replaced by $x_{y}$. 
$\mathscr{R}$ is defined by its row and column squares $R_{1}$ and $R_{2}$. Each $\hat{R}_{\alpha}$ is a $(2 m-1) \times(2 m-1)$ array whose entries are $(2 n-1) \times(2 n-1)$ blocks:

$$
\begin{aligned}
& \text { the }(i, i) \text { block of } \hat{R}_{\alpha} \text { is } N_{x}(i), \\
& \text { the }(i, j) \text { block of } \hat{R}_{\alpha} \text { is } L_{\alpha}\left(a_{i j}^{\alpha}\right) \text { if } i<j, \\
& \text { the }(i, j) \text { block of } \hat{R}_{\alpha} \text { is } L_{\alpha}\left(a_{i j}^{\alpha}\right)^{T} \text { if } i>j .
\end{aligned}
$$

The last row and column of $R_{\alpha}$ are $(1,2, \cdots,(2 m-1)(2 n-1), 0)$. One sees immediately that the $R_{\alpha}$ are symmetric Latin squares with constant diagonal 0 .

Assume that $k$ and $l$ are non-zero. If the entry $\left(k_{i}, l_{i}\right)$ occurs in $\left(\hat{R}_{1}, \hat{R}_{2}\right)$ then it occurs in the block $\left(\hat{N}_{1}(i), \hat{N}_{2}(i)\right)$ at the place where $(k, l)$ occurs in $\left(\hat{N}_{1}, \hat{N}_{2}\right)$; since $\mathscr{N}$ is a Room square, $(k, l)$ can arise at most once above the diagonal, and if also $\mathscr{N}$ is skew-type, $(l, k)$ cannot also arise above the diagonal in $\left(\hat{N}_{1}, \hat{N}_{2}\right)$. So $\left(k_{i}, l_{i}\right)$ can occur at most once above the diagonal in $\left(\hat{R}_{1}, \hat{R}_{2}\right)$; if $\mathscr{N}$ is skew-type, $\left(l_{i}, k_{i}\right)$ and $\left(k_{i}, l_{i}\right)$ cannot both occur. (We need not distinguish the case $k_{i}=l_{i}$, since this pair does not arise in $\left(\hat{R}_{1}, \hat{R}_{2}\right)$.) If the entry $\left(k_{i}, l_{j}\right)$ occurs in $\left(\hat{R}_{1}, \hat{R}_{2}\right)$, $i \neq j$, it occurs above the diagonal in the block $\left(L_{1}(i), L_{2}(j)\right)$, which appears at most once since $\mathscr{M}$ is a Room square; $\left(k_{i}, l_{j}\right)$ occurs only once in $\left(L_{1}(i), L_{2}(j)\right)$ since $L_{1}$ and $L_{2}$ are orthogonal. If further $\mathscr{M}$ is skew-type then $\left(L_{1}(i), L_{2}(j)\right)$ and $\left(L_{1}(j), L_{2}(i)\right)$ cannot both be in $\left(\hat{R}_{1}, \hat{R}_{2}\right)$, so $\left(k_{i}, l_{j}\right)$ and $\left(l_{j}, k_{i}\right)$ cannot both occur. The last column of $\left(R_{1}, R_{2}\right)$ contains the pairs $(i, i)$ once each. So, from Theorem 1 , $R_{1}$ and $R_{2}$ define a (standardized) Room square $\mathscr{R}$ of the required order. If $\mathscr{M}$ and $\mathscr{N}$ are skew-type then $\mathscr{R}$ is skew-type.

COROLlaRY 5. If $2 n-1=\prod p_{i}^{r_{i}}$, where each $p_{i}$ is a prime congruent to 3 modulo 4 and no $p_{i}^{r_{i}}$ is $3,3^{2}$, or $3^{4}$, then there is a skew-type Room square of order $2 n$ and consequently a Room squre of order $4 n$.

This Corollary simply combines Theorems 2 and 4 and Corollary 3. It gives ten new Room square orders less than 500, including 56,88 and 96 . If there is a skew-type Room square of order 10 , then we need only bar the case $p_{i}^{r_{i}}=3$ in Corollary 5 .

\section{References}

[1] R. W. Archbold and N. L. Johnson, 'A construction for Room's squares and an application in experimental design', Ann. Math. Statist. 29 (1958), 219-225.

[2] R. C. Bose, E. T. Parker and S. S. Shrikhande, 'Further results on the construction of mutually orthogonal Latin squares and the falsity of Euler's conjecture', Canad. J. Math. 12 (1960), 189-203.

[3] R. H. Bruck, "What is a loop?", Studies in Modern Algebra (ed. A. A. Albert; Mathematical Association of America, 1963), 59-99.

[4] R. C. Mullin and E. Nemeth, 'An existence theorem for Room squares', Canad. Math. Bull. 12 (1969), 493-497. 
[5] R. C. Mullin and E. Nemeth, 'On furnishing Room squares', J. Combinatorial Theory 7 (1969), 266-272.

[6] T. G. Room, 'A new type of magic square’, Math. Gazette 39 (1955), 307.

[7] H. J. Ryser, Combinatorial Mathematics (Mathematical Association of America, 1963).

[8] R. G. Stanton and R. C. Mullin, 'Construction of Room squares', Ann. Math. Statist. 39 (1968), 1540-1548.

[9] R. G. Stanton and J. D. Horton, Composition of Room Squares, Combinatorial Theory and its Applications (Colloquia Mathematica Societas Janos Bolyai 4) (North Holland, Amsterdam, 1970), 1013-1021.

Faculty of Mathematics

University of Newcastle

New South Wales, 2308

Australia 